\title{
Teorías en torno a la globalización y sus impliçaciones para el desarrollo económico latinoamericano
}

Theories concerning the Globalization and his Implications for the Economic Latin-American Development

\section{Resumen}

A partir de la revisión de los planteamientos, a veces discrepantes, de tres de sus autores contemporáneos más reconocidos, este trabajo propone hacer una reflexión alrededor de lo que significa el complejo fenómeno conocido coloquialmente como globalización, los rasgos que incorpora y algunas de sus implicaciones para el desarrollo económico en América Latina. Como parte de sus conclusiones se plantea que una de las variables explicativas más importantes para comprender el lento y dispar desarrollo en la región, es la generalizada ausencia de Estados, gobiernos y administraciones públicas eficientes y legítimos. Es decir, la globalización y las políticas pro mercado distan de ser las responsables de todos los males que aquejan a Latinoamérica, siendo necesario además para transitar hacia otros estadios de desarrollo, que los Estados y los mercados trabajen en asociación.

$$
\begin{array}{cl}
\text { Palabras clave: } \\
\text { • } & \text { Globalización } \\
\text { • } & \text { Desarrollo } \\
& \text { Latinoamérica }
\end{array}
$$

JEL: F01, O19, O54

\author{
Jaime Torres Fragoso*
}

\section{Abstract}

This paper proposes a reappraisal of the complex phenomenon known colloquially as globalization. This reappraisal is based on a review of the approaches put forward by three leading contemporary experts in the field. It also examines the most important elements of the globalization process and its effects on economic development in Latin America. These expert's findings suggest that one of the explanatory variables in the slow and uneven rate of development in Latin America is the general absence of States, governments and public administrations that lack legitimacy and efficiency. In other words, the process of globalization and market economy policies are not directly responsible for all of Latin America's woes. Furthermore States and markets should work in partnership to move towards new phases of development.

\section{Keywords: \\ - Globalization \\ - Development \\ - Latin America}

\section{Introducción}

Uno de los temas más controvertidos desde hace varios años es el referente a las bondades y desventajas del fenómeno conocido como globalización, asunto con el que de alguna manera todos tienen que ver. La polémica inicia desde su misma definición, en torno a la cual no existe consenso dado el abanico de opciones que pueden ser incorporadas en ella. ¿Se trata de una versión corregida y aumentada del modelo tradicional de producción capitalista transformado por la modernidad? ¿La globalización Implica fundamentalmente la movilidad financiera y de capitales, de productos y mercancías, así como de imágenes e información, o repercute también en nuestros arreglos sociales e institucionales, en nuestra cultura, tradiciones y valores? ¿Se encuentra en una fase embrionaria o vive una última etapa histórica? ¿Sus efectos negativos colaterales y riesgos superan a sus oportunidades y beneficios para la humanidad en general y para 
América Latina en lo particular? Todas ellas son algunas de las interrogantes planteadas en la búsqueda de esa definición.

En ese sentido, este trabajo analizará en primer término tres de los retos del Estado contemporáneo en Latinoamérica: el desarrollo, económico (tomando como referente el inicio en la aplicación de políticas macroeconómicas denominadas como neoliberales), la valoración de la sociedad respecto a su situación económica y a la sensación de progreso, además de los indicadores sobre la percepción de la corrupción en nuestro subcontinente. Con la intención de darle sustento teórico a esos retos, posteriormente se revisarán tres posiciones teóricas encontradas en la bibliografía especializada sobre este fenómeno y que expresan algunas de las contradicciones señaladas arriba: Imperio e Imperialismo, de Atilio A. Boron; El malestar en la globalización, de Joseph E. Stiglitz; y Reformas económicas, globalización y gobernabilidad en América Latina, de Ricardo Ffrench-Davis. Se escogieron estos textos dado que expresan posiciones distintas y en varias ocasiones encontradas en torno al fenómeno. Para caracterizarlos de alguna manera, el trabajo de Boron presenta una postura en contra de la globalización y el mercado, a los cuales responsabiliza de varios de los problemas económicos y sociales de nuestra región. El texto de Stiglitz maneja una postura que podríamos definir como intermedia, indicando que si bien la globalización constituye una variable explicativa para el entendimiento de los rezagos y el inadecuado desarrollo de Latinoamérica, el factor fundamental para acceder a una realidad diferente lo representa la constitución de Estados y arreglos institucionales internos sólidos y eficaces. Por su parte, el trabajo de Ffrench-Davis resta relevancia a la influencia de la globalización y encuentra como factor fundamental la capacidad organizacional de nuestros Estados y gobiernos en asuntos económicos y administrativos; de alguna forma este autor desmitifica los impactos de la globalización en nuestras vidas.

Esta investigación asume que en torno a esta y a cualquier temática no existen teorías "vencedoras y perdedoras", es decir, las que se puedan asumir como únicas y poseedoras de la verdad absoluta, que vendrían representando una especie de panacea, o bien aquellas que carezcan de valor y puedan menospreciarse. Por el contrario y como es natural, se considera que entre el conjunto de ideas encontradas en los textos revisados y en los muchos más disponibles con relación al tema, de hechura ya sea regional o proveniente del resto del mundo, se encuentra parte de la verdad.

En tal sentido, la pregunta central del trabajo que motivó emprender esta investigación es la siguiente: ¿La globalización es una variable exegética fun- 
damental o existen otros elementos sustantivos para comprender el grado de desarrollo social y económico experimentado en Latinoamérica? De acuerdo a lo anterior, la hipótesis central de esta investigación es que si bien la globalización podría explicar en cierta medida las desventajas económicas y sociales de América Latina, gran parte de los apuros del subcontinente obedecen a una serie de presencias y ausencias. Presencias generalizadas de Estados, aparatos de gobierno y administraciones públicas ineficientes, con insuficiente grado de profesionalismo y, lo que es más grave, deshonestos. ${ }^{1}$ Ausencias de, entre otras cosas, arreglos institucionales más racionales que permitan generar mejores políticas públicas.

\section{Los retos del Estado contemporáneo en América Latina}

\section{El desarrollo económico}

Tal como lo sintetiza Rodríguez (1997), para diversos investigadores el ingreso por habitante es uno de los indicadores más utilizados para medir el nivel de desarrollo económico. Con base en ello, observamos, de acuerdo a la Tabla I, que el desarrollo económico en Latinoamérica muestra todavía resultados desventajosos. No obstante, se debe resaltar que en 1983, año que en lo general coincide con el inicio de la aplicación de modelos de economía de mercado en la región, la situación era claramente peor, particularmente en Bolivia, El Salvador, Guatemala, Honduras, Nicaragua y Perú. En 1993 nuestros países muestran un muy interesante avance generalizado en el PIB per cápita, donde destacan los casos de Argentina, Chile, Costa Rica, México y Uruguay; siendo las excepciones los casos de Bolivia, Cuba, Ecuador, Guatemala, Honduras, Nicaragua (que experimentó una situación alarmante) y Venezuela.

\footnotetext{
${ }^{1}$ Por supuesto que esto es dicho en términos muy generales y reconociendo que existen varias excepciones, es decir, casos de gobiernos aptos y honestos en América Latina.Además, con las reformas administrativas implantadas en años recientes, las cuales han incorporado varios de los postulados de la nueva gerencia pública, auspiciada por organismos como el CLAD, hemos experimentado una mejora sustancial de nuestras administraciones públicas.
} 


\section{PIB per cápita en América Latina (precios en dólares estadounidenses)}

\begin{tabular}{|c|c|c|c|c|}
\hline País & 1983 & 1993 & 2003 & 2013 \\
\hline Argentina & $3,532.50$ & $6,971.10$ & $4,134.50$ & $14,715.20$ \\
\hline Bolivia & 941.00 & 786.40 & 896.40 & $2,867.20$ \\
\hline Brasil & $1,558.40$ & $2,791.50$ & $3,039.70$ & $11,208.10$ \\
\hline Chile & $1,685.20$ & $3,416.90$ & $4,866.30$ & $15,732.30$ \\
\hline Colombia & $1,344.60$ & $1,582.60$ & $2,261.30$ & $7,831.20$ \\
\hline Costa Rica & $1,557.10$ & $2,909.80$ & $4,199.70$ & $10,184.60$ \\
\hline Cuba & $2,226.10$ & $2,066.90$ & $3,192.40$ & $\star 6051.2$ \\
\hline Ecuador & $2,011.10$ & $1,747.70$ & $2,442.30$ & $6,002.90$ \\
\hline El Salvador & 719.70 & $1,239.50$ & $2,495.60$ & $3,826.10$ \\
\hline Guatemala & $1,199.70$ & $1,195.90$ & $1,816.90$ & $3,477.90$ \\
\hline Honduras & 771.50 & 654.50 & $1,228.10$ & $2,290.80$ \\
\hline México & $1,988.10$ & $5,498.50$ & $6,601.10$ & $10,307.30$ \\
\hline Nicaragua & 779.90 & 394.70 & $1,000.90$ & $1,851.10$ \\
\hline Panamá & $2,291.40$ & $2,741.10$ & $3,990.70$ & $11,036.80$ \\
\hline Paraguay & $1,499.70$ & $1,582.40$ & $1,159.40$ & $4,264.70$ \\
\hline Perú & 905.80 & $1,483.40$ & $2,180.50$ & $6,661.60$ \\
\hline Uruguay & $1,716.00$ & $4,720.80$ & $3,622.30$ & $16,350.70$ \\
\hline Venezuela & $4,784.20$ & $2,746.10$ & $3,241.50$ & $14,414.80$ \\
\hline
\end{tabular}

$\star$ : Se refiere al año 2011.

Fuente: Banco Mundial (2015).

Entre 1993 y 2003 la región registró avances muy modestos, donde destacan Chile, Costa Rica, Cuba, El Salvador, Guatemala, Honduras, Nicaragua y Panamá en cuanto al crecimiento relativo de este indicador; mientras que, por el contrario, Argentina, Paraguay y Uruguay observaron evidentes retrocesos. Por el contrario, en la década que va de 2003 a 2013 todos los países latinoamericanos presentaron un crecimiento muy evidente (aunque por desgracia, como en la mayoría de las naciones centroamericanas, claramente insuficiente) y en algunos casos, como los de Argentina, Brasil, Chile, Costa Rica, México, Panamá, Uruguay y Venezuela, los indicadores muestran cifras totales muy estimables, ubicándose entre los 10 mil y 16 mil dólares.

A pesar de los buenos números que la región presenta en los últimos años, los cuales en alguna medida se explican por la aplicación de estrategias que el modelo de economía de mercado incorpora, su posición relativa, respecto a 
países desarrollados o en vías de convertirse en eso, es aún desfavorable como antes se señaló. Así, tenemos que en ese 2013 Alemania alcanzó un PIB per cápita de 46286.6 dólares estadounidenses, Estados Unidos registró 53 042.0, Singapur llegó a 55 182.5, mientras que el de Corea del Sur fue de 25977.0 (Banco Mundial, 2015). Lo acontecido en Corea supone una interesante lección, ya que el ingreso promedio por habitante de este país en 1983 era de 2 268.2 dólares, comparable al promedio de Latinoamérica, mientras que en la actualidad presenta un registro muy superior al de Uruguay o Chile, las naciones líderes en ese rubro en esta región. ${ }^{2}$ Además de ese rezago, hay que recordar que la región, de acuerdo a numerosos estudios, es la más desigual del planeta (véase, por ejemplo, Kliksberg, 2007), por lo que hay que considerar que el PIB per cápita tiene un evidente sesgo por la inequitativa distribución de la riqueza en estos países. ${ }^{3}$ Independientemente de ello, la evidencia sugiere que en Latinoamérica se advierten importantes esfuerzos en materia de desarrollo económico y social, los cuales no son despreciables pero sí insuficientes como ya se advirtió.

\section{Percepción de la situación económica y sensación de progreso}

Lo analizado en el anterior apartado coincide en términos generales con el estudio de Latinobarómetro (2010), en el que se encuentra que (seguramente sustentada en lo acontecido en la última década), la opinión de la ciudadanía respecto a su situación económica es favorable. Así y como se presenta en el cuadro II, tenemos que en todo el subcontinente sólo 35\% de la ciudadanía piensa que su realidad económica es mala o muy mala, cifra que en Brasil y Uruguay es únicamente de 14\%, y en Chile de 16\%. Mientras que El Salvador, Guatemala, Honduras, México (explicable por ser un perfecto ejemplo de distribución desigual del ingreso) y Nicaragua, son los casos donde la mayoría o una porción cercana a la mitad de la población percibe una situación económica negativa.

\footnotetext{
${ }^{2}$ Además de fuertes inversiones en ciencia y tecnología, la República de Corea le ha apostado a una economía de mercado dominada por poderosas compañías conglomeradas llamadas chaebol, las que se han beneficiado por un esquema de asociación entre el gobierno y los conglomerados industriales, pero sin permitir que éstos absorban a las pequeñas empresas (Koontz, Weihrich y Cannice, 2008).

${ }^{3}$ De acuerdo a un reciente estudio de la Corporación Latinobarómetro (2010), únicamente 21\% de la población en América Latina considera que la distribución de la riqueza es justa o muy justa. Además, $60 \%$ está de acuerdo o muy de acuerdo en que las decisiones del gobierno buscan privilegiar a unos pocos.
} 
Por otra parte, la misma tabla indica un resultado menos positivo en lo que respecta a la sensación de progreso. A nivel global, sólo 39\% de la población consideró que observaba progreso económico en sus países, destacando nuevamente los casos de Brasil, Chile, Panamá y Uruguay (cuatro de los seis países con mayor ingreso por habitante en la región), en los que al menos la mitad de la población percibió tal progreso. En el extremo opuesto se ubican Costa Rica (un caso que sorprende, junto a Argentina), El Salvador, Guatemala, Honduras (los últimos como casos extremos) y México, donde ni siquiera un tercio de la población manifiesta sentir progreso económico.

\section{Percepción de la situación y progreso económica en América Latina}

\begin{tabular}{|c|c|c|}
\hline País & Situación económica ${ }^{\star}$ & Sensación de progreso $\star \star$ \\
\hline Argentina & 36 & 33 \\
\hline Bolivia & 31 & 34 \\
\hline Brasil & 14 & 68 \\
\hline Chile & 16 & 55 \\
\hline Colombia & 29 & 39 \\
\hline Costa Rica & 24 & 31 \\
\hline Ecuador & 24 & 49 \\
\hline El Salvador & 52 & 24 \\
\hline Guatemala & 56 & 14 \\
\hline Honduras & 58 & 10 \\
\hline México & 49 & 24 \\
\hline Nicaragua & 48 & 39 \\
\hline Panamá & 22 & 67 \\
\hline Paraguay & 40 & 38 \\
\hline Perú & 29 & 40 \\
\hline Uruguay & 14 & 50 \\
\hline Venezuela & 33 & 40 \\
\hline Latinoamérica & 35 & 39 \\
\hline
\end{tabular}

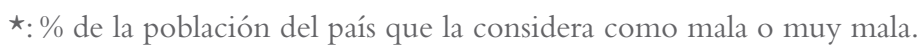

$\star \star$ : \% que considera que la situación económica está progresando.

Fuente: Elaboración propia con base en Corporación Latinobarómetro (2010). 
Como datos complementarios conviene decir que 38\% de los ciudadanos de América Latina ubican como problemas graves los asuntos económicos, la pobreza y el desempleo, además de que 58\% está de acuerdo o muy de acuerdo en que la economía de mercado es el único sistema con el que se puede llegar a ser un país desarrollado (Latinobarómetro, 2010).

La percepción de corrupción en la región

Existe suficiente evidencia para aseverar que el desarrollo económico y social de las naciones se correlaciona positivamente con la ausencia de corrupción y viceversa. Dicho de otra manera, las naciones más desarrolladas, como las escandinavas, presentan los menores índices de corrupción, a la vez que las más pobres muestran niveles de corrupción muy preocupantes. América Latina no escapa a esa dinámica y, por desgracia, la gran mayoría de las naciones que la integran manifiestan severos problemas de corrupción.

Con base en el cuadro III, referente al Índice de Percepción de la Corrupción (IPC) 2014, se observa que, con las honrosas excepciones de Chile y Uruguay (precisamente los países con mayor desarrollo económico de la región, por lo que la correlación antes mencionada se confirma), y en cierta forma de Costa Rica, las situación en las demás naciones en materia de corrupción es reprobatoria y muy preocupante. Si bien los ejemplos más notorios son los de Venezuela, que obtiene 19 puntos de 100 posibles, ${ }^{4}$ que lo posicionan en el lugar 161 de 174 países participantes en el estudio; Paraguay, con 24 puntos y la posición 150; Nicaragua, que obtiene 28 unidades que lo sitúan en el lugar 133; además de Honduras, que recibe 29 puntos para ubicarse en la posición 126. En sentido opuesto, las 73 unidades que reciben tanto Chile como Uruguay les permiten ocupar el lugar 21, en niveles similares a los de alguna nación desarrollada.

\footnotetext{
${ }^{4}$ La puntuación del IPC indica el grado de corrupción en el sector público de acuerdo a la percepción de empresarios y analistas del país.Va de 100 (percepción de nula corrupción) a 0 (percepción de total corrupción).
} 


\section{Índice de Percepción de la Corrupción 2014 en} América Latina

\begin{tabular}{|l|c|c|}
\hline \multicolumn{1}{|c|}{ País } & Puntuación & Posición \\
\hline Argentina & 34 & 107 \\
\hline Bolivia & 35 & 103 \\
\hline Brasil & 43 & 69 \\
\hline Chile & 73 & 21 \\
\hline Colombia & 37 & 94 \\
\hline Costa Rica & 54 & 47 \\
\hline Cuba & 46 & 63 \\
\hline Ecuador & 33 & 110 \\
\hline El Salvador & 39 & 80 \\
\hline Guatemala & 32 & 115 \\
\hline Honduras & 29 & 126 \\
\hline México & 35 & 103 \\
\hline Nicaragua & 28 & 133 \\
\hline Panamá & 37 & 94 \\
\hline Paraguay & 24 & 150 \\
\hline Perú & 38 & 85 \\
\hline Uruguay & 73 & 21 \\
\hline Venezuela & 19 & 161 \\
\hline Fuente: & & \\
\hline
\end{tabular}

Fuente: Adaptada de Transparencia Internacional España (2015)

Para contrastar estos datos, mencionemos que Dinamarca, con sus 92 puntos se ubicó en el lugar número uno (el país menos corrupto del mundo); Nueva Zelanda, 91 puntos y lugar número dos; y Finlandia, 89 puntos y posición número tres. Un poco atrás aparecen Singapur, 84 unidades y séptimo lugar; Canadá, 81 puntos y posición diez; Alemania, 79 unidades y lugar doce; Estados Unidos, 74 puntos (solamente uno más que Chile y Uruguay) y lugar diecisiete; asimismo, Corea del Sur obtuvo 55 puntos que lo ubicaron en la posición 43. En el extremo contrario, los países que ocuparon los últimos lugares en el estudio fueron Somalia y Corea del Norte, que obtuvieron 8 puntos y la posición 174; Sudán, con 11 puntos y el lugar 173; además de Afganistán, que recibió 12 puntos y la posición 172. En el hemisferio occidental Venezuela y Haití, con idénticos resultados, se ubicaron como las naciones con los más bajos puntajes. 
Economía Informa núm. 391 marzo - abril • 2015

\title{
2. Diversas posturas teóricas respecto a la globalización
}

\author{
Atilio A. Boron
}

Como se indicó anteriormente, la posición de Boron (2002) es totalmente adversa a la idea de la globalización, el capitalismo y una de las que para muchos, incluyendo a Boron, representa una de sus manifestaciones: el imperialismo. ${ }^{5}$ De acuerdo a Boron, la globalización representa la teoría neoliberal de pensamiento único, una ideología económica dominante divulgada por el Fondo Monetario Internacional (FмI), el Banco Mundial (BM) y otros organismos hegemónicos, como la Organización Mundial de Comercio (OMC). Para él, el imperialismo contemporáneo ha cambiado respecto al de años atrás, originando una economía global donde todos somos interdependientes, oprimiendo a pueblos y naciones, y sembrando a su paso, dolor, destrucción y muerte. Para Boron, el imperialismo no es un rasgo accesorio, sino que representa una nueva etapa en el desarrollo del capitalismo, determinada por la concentración del capital, el predominio de los monopolios, el rol protagónico del capital financiero, la exportación de capitales y el reparto del mundo en diversas áreas de influencia, de acuerdo a lo anterior, indica Boron, la globalización vino a consolidar la dominación imperialista y profundizó la sumisión de los capitalismos periféricos, incluidos los latinoamericanos.

En este orden, algunos de los más importantes impactos desfavorables del dominio imperialista serían, a su entender, el sistemático genocidio que el imperialismo practica a diario en los países de la periferia capitalista, la regresión social y la desciudadanización, la destrucción del medio ambiente, el desplome de los regímenes democráticos maniatados por la tiranía de los mercados y la acometida militar que emprendieran los Estados Unidos a partir de la administración de Bush como respuesta a los atentados cometidos a las Torres Gemelas y el Pentágono. Además de reconocer a la onU como una organización que se mueve en el gatopardismo (no es lo que aparenta ser, dice el autor), elitista, burocrática y destinada a defender los intereses imperialistas, particularmente los de Estados Unidos.

\footnotetext{
${ }^{5}$ Esta idea no es compartida por el autor de este ensayo ni por Rob Kroes, quien plantea: "Puede que el mundo se esté convirtiendo en una aldea global, pero esto no quiere decir que sus habitantes se conviertan en ciudadanos del mundo. Si hay alguna tendencia, esta va en dirección contraria. El número de estados independientes que organizan a los habitantes del mundo en relación a su ciudadanía y nacionalidad ha aumentado sin cesar, sobre todo en el siglo XIX. Por supuesto, ésta crece año por año con cada imperio que se derrumba y con cada grupo étnico que se separa y establece su propio Estado" (Kroes, 2000: 120).
} 
En una visión que se antoja un tanto aventurada, Boron indica que ante tal realidad, las masas oprimidas y explotadas del mundo son convocadas para una batalla final contra un régimen de relaciones globales, ${ }^{6}$ y que incluso existe un lenguaje y una comprensión común entre las diferentes luchas que se emprenden a lo largo de todo el orbe en contra de la dictadura del capital. No obstante, este autor reconoce algunas de las bondades de la modernidad: las libertades individuales, la relativa igualdad alcanzada en los terrenos económicos, políticos y sociales dentro de los capitalismos avanzados, el sufragio universal y la democracia, así como el progreso económico. ${ }^{7}$

Otro nivel de análisis interesante en el trabajo de Boron lo constituye lo referente a la eficiencia de las empresas públicas. Para él, cosa en la que coincidimos totalmente, es falsa la afirmación de que éstas son necesariamente ineficientes o productoras de bienes o servicios de mala calidad, y que a la vez las empresas privadas satisfacen plenamente los requerimientos de los consumidores. Asimismo, señala que para el caso latinoamericano, los promotores de la política neoliberal (cuyos arquetipos identifica en Carlos Menem, Carlos Salinas de Gortari y Alberto Fujimori) han demostrado su incapacidad para sostenerse en el poder. ${ }^{8}$

Asimismo, a algunas iniciativas que pretenden la integración económica de la región, particularmente al Plan Puebla-Panamá, las ubica como otra iniciativa imperialista. Desde su perspectiva, dicho plan se signó para solucionar el conflicto originado por el levantamiento armado en Chiapas y, a la vez, establecer una cabeza de playa en la mayor reserva acuífera de México con la intención de abastecer de agua al sur de California. ${ }^{9}$ En suma, Boron plantea

\footnotetext{
${ }^{6}$ No deja de causar sorpresa tal propuesta, ya que incluso se insinúa una conspiración militar. En realidad, en caso de que tal propuesta tuviera viabilidad, sería muy difícil pensar que esa desnivelada batalla se podría ganar. Por el contrario, creemos que un camino más útil y menos costoso, en todos sentidos, es apelar a las posibilidades de la negociación y del establecimiento de acuerdos en diferentes ámbitos.

${ }^{7}$ Es interesante (y pareciera que incluso contradictorio en cierta medida) el reconocimiento de Boron de algunos de los efectos positivos de la modernidad, cuya vertiente económica generalmente se asocia a modelos de mercado, más aún que acepte la igualdad lograda en los sistemas capitalistas más desarrollados.

${ }^{8}$ Esta es una afirmación que pareciera un tanto arriesgada, al menos para el caso mexicano. En México, después de Salinas de Gortari, Ernesto Zedillo (surgidos del mismo partido político, el PRI) aplicó el mismo tipo de políticas pro mercado. Incluso con el cambio de régimen y con el arribo deVicente Fox y de otro partido a la Presidencia, el pan, se aplicó, en términos generales, el mismo modelo económico "neoliberal"; lo mismo ocurrió en el gobierno de Felipe Calderón y, por supuesto, con el regreso del PRI en 2012.

${ }_{9}$ Con el paso del tiempo se observa que esa estrategia (que incluso cambió su nombre a Proyecto Mesoamérica) no pretendía ni una cosa ni la otra. Sería una sobre simplificación de la realidad pretender solucionar el complejo conflicto chiapaneco, plenamente vigente en varios problemas y
} 
que la opresión imperialista continúa su accionar, representada, más que por un orden internacional, por el poderío económico, político y militar de los Estados Unidos.

\section{Joseph E. Stiglitz}

Como se indicó en la introducción, el trabajo de Stiglitz (2002) representa una posición intermedia, en la que le otorga validez explicativa respecto al nivel de los indicadores sociales experimentados en Latinoamérica, tanto a los fenómenos ligados a la globalización como a la capacidad (o incapacidad) institucional y organizacional de estos Estados. Parte de reconocer que en amplios sectores de la población mundial, particularmente en los más pobres, los efectos de la globalización no han sido adecuados, así como en tópicos como la ecología y la estabilidad económica internacional; situación que también aplica para los países que transitaron del comunismo a la economía de mercado. No obstante, agrega, la globalización también ha producido estimables beneficios, como las oportunidades del comercio, los accesos a mercados y a la tecnología, en la salud, en la democracia y justicia social.

Para este autor, gran parte de los problemas aparejados con la globalización están vinculados a un mal accionar de las instituciones económicas internacionales antes referidas (FMI, BM у OMC). A su parecer, éstas han favorecido los intereses de los países industrializados (y dentro de éstos, aún más a las potencias hegemónicas) sobre los de las naciones subdesarrolladas, debido a que han enfocado la globalización desde una perspectiva unidireccional, siguiendo modelos muy específicos en materia económica y social. Al respecto, Stiglitz observa una necesidad tanto de realizar cambios a fondo en la arquitectura financiera global, como de replantear la globalización a partir del rediseño de los organismos económicos internacionales.

Stiglitz igualmente reconoce diferentes tipos de modelos y sistemas de mercado, por ejemplo, indica que en Suecia el Estado asume responsabilidades muy altas en la promoción del bienestar social, proporcionando servicios de salud pública, seguros de desempleo y pensiones de mucho mayor calidad que las que se tienen en Estados Unidos, cuyo modelo, si bien ha funcionado adecuadamente para muchos, sería inaceptable para la mayoría de los suecos. Por

deudas sociales en ese estado, mediante esa estrategia. Por otra parte, además de que México (tan sumiso en otras situaciones frente a su vecino del norte) ha mantenido históricamente férreas disputas en torno a la propiedad del agua del Río Bravo (frontera natural) con los Estados Unidos, resulta inviable económicamente tal acción. 
consiguiente, agrega Stiglitz, el Estado cumple un rol esencial no sólo para atenuar esos fallos de mercado comentados, sino también para hacer que la justicia social prevalezca. Agrega que en países donde esas condiciones se han presentado, los Estados suministraron una educación de alto nivel a todos sus habitantes y ofertaron la mayoría de la infraestructura; impusieron un buen marco legal (necesario para que los mercados operen correctamente); impusieron regulaciones al sector financiero; establecieron una red de seguridad para los pobres; además de que promovieron y desarrollaron la tecnología en diversos ámbitos. En síntesis, de acuerdo a esta perspectiva, el Estado cumple una función estratégica para que cualquier sociedad y economía se desarrollen con eficacia y sentido humano.

Por otra parte y siguiendo al mismo autor, a pesar de que las intervenciones del Estado puedan incrementar la eficiencia de los mercados, los partidarios del fundamentalismo de mercado argumentan que las ineficiencias de los mercados son relativamente pequeñas y que las ineficiencias del Estado son comparativamente grandes, culpando a éste de situaciones que el sentido común señala que son fallas de mercado (agrega Stiglitz que Adam Smith era más consciente de las limitaciones del mercado que sus propios seguidores). No obstante, lo que busca Stiglitz es una perspectiva equilibrada del papel del Estado, donde se reconozcan sus limitaciones y fallas, pero también las de los mercados, que pretende acercar a ambos para que actúen en forma conjunta como socios. $^{10}$

Stiglitz añade que el malestar con la globalización obedece no tanto al dominio de la economía sobre los demás estadios de la realización humana, sino al predominio de una visión muy específica de la economía, vinculada al fundamentalismo de mercado (la creencia de que existe un conjunto único de políticas que es el correcto), impuesto por los organismos financieros internacionales.

Para este investigador, el cambio requerido más importante para que la globalización funcione como debiera, es un cambio en la gobernanza, lo que implicaría cambios profundos en el sistema financiero internacional, así como transformaciones drásticas en la lógica sobre la que vienen actuando el FMI, el вм y la omc, de tal forma que no sean sólo las voces de los ministros de Hacienda y de Comercio las que se oigan en ellas. Asimismo, se requiere que estas instituciones actúen con mucha mayor transparencia para garantizar que puedan responder mejor a los requerimientos de los sectores más vulnerables y desprotegidos, del medio ambiente, y de los múltiples problemas políticos

${ }^{10}$ Cabe aclarar que en realidad este planteamiento no es del todo novedoso, Osborne y Gaebler propusieron antes ese mismo concepto en su obra La Reinvención del Gobierno, en 1992. 
y sociales de carácter coyuntural y estructural experimentados en prácticamente todo el orbe. En suma, el autor plantea tanto una ambiciosa reforma del FMI y del sistema financiero global que incorpore, entre otros elementos, la aceptación de los peligros de la liberalización de los mercados de capitales, reformas sobre quiebras y moratorias, el otorgar menos recursos a los salvamentos, mejorar la regulación bancaria y una mejor gestión del riesgo. Además, se requiere que el BM gestione ayudas específicas y la condonación de la deuda para los países en desarrollo, y que la OMC equilibre las agendas comerciales y laborales.

A manera de resumen, lo que está planteando Stiglitz es el establecimiento de una ruta que permita transitar hacia una globalización con un rostro más humano, expresión que él acuña con mucho tino. Por ejemplo, señala que Europa defiende sus políticas agrícolas no sólo en función de intereses particulares, sino también para preservar las tradiciones rurales (como lo acontecido en la industria del vino). Agrega que la globalización, tal como generalmente es defendida, parece sustituir a las antiguas dictaduras de las élites nacionales por las nuevas dictaduras de las finanzas internacionales. ${ }^{11}$ Stiglitz agrega que la adopción de este modelo con rostro más humano es necesario ante el malestar justificado que persiste en muchas latitudes con la globalización, a pesar de que ésta, con las consideraciones a que haya lugar, puede ser vista como un acontecimiento positivo. Por ejemplo, agrega este autor, la generalización de las ideas en torno a la democracia ha transformado la forma de pensar de las personas; la globalización ha permitido a millones de individuos acceder a mejores niveles de vida; mientras que la globalización económica ha beneficiado a los países que se han insertado en ella, abriendo nuevos mercados para sus exportaciones y mecanismos para la inversión extranjera.

Otra observación fundamental de Stiglitz y que representa una lección para América Latina, es que los países que más provecho han obtenido de la globalización han sido los que se responsabilizaron de su propio destino y se dieron cuenta del importante rol que puede cumplir el Estado en el desarrollo, sin apostar a la idea de un mercado autorregulado que solucione todos nuestros problemas. Lo anterior sin dejar de reconocer que, al mismo tiempo, para millones de personas la globalización no ha funcionado bien. Esto se pude explicar parcialmente, continúa Stiglitz, por una rápida liberalización de

\footnotetext{
${ }^{11}$ Ese esquema pareciera común en América Latina, donde el fin de las dictaduras militares dio pauta a la apertura política y económica, a raíz de la cual se adoptaron modelos de mercado en forma irreflexiva y $\sin$ los ajustes y controles necesarios.
} 
los mercados de capitales sin una buena regulación (situación que como ya se señaló antes, refleja parte de lo acontecido en la región).

Por consiguiente, apunta el mismo autor, los países en desarrollo, incluyendo los latinoamericanos, deben asumir la responsabilidad de su propio bienestar. Para esto requieren como condiciones necesarias Estados eficaces, poderes judiciales fuertes e independientes, sistemas políticos democráticos, apertura, transparencia y el combate efectivo a la corrupción. ${ }^{12}$ Como bien lo indica Stiglitz, el desarrollo no significa ayudar a un sector de la población o de la industria a enriquecerse a expensas de otros, ni que en las ciudades los sectores más poderosos económicamente dispongan de productos y bienes de marcas importadas de lujo. ${ }^{13}$ En cambio, lo que sí significa es lograr una transformación de la sociedad, mejorar las condiciones de todos, principalmente de los más pobres, y permitir que toda la población tenga la oportunidad de mejorar su nivel de vida y acceder a servicios sociales básicos como la salud y la educación, cuya oferta es responsabilidad absoluta del Estado. ${ }^{14}$

\section{Ricardo Ffrench-Davis}

El trabajo de Ffrench-Davis marca otra posición que luce muy sensata, en la que los factores explicativos de la realidad política, económica y social de Latinoamérica se buscan y encuentran en la capacidad o incapacidad (ya sea por ineptitud o apatía) interna o local, de parte de nuestros gobiernos, para generar políticas adecuadas en esos mismos ámbitos. Para Davis, el fenómeno de la globalización y la supuesta adopción del modelo de economía de mercado, constituye una variable explicativa intrascendente o, si acaso, de orden menor.

La argumentación de este investigador es que, erróneamente, a la globalización se le pretende ver como un dato inmodificable, como una variable contextual fija. Por el contrario, agrega Ffrench-Davis, la globalización se construye a partir de políticas nacionales, aún en países pequeños, pues

\footnotetext{
${ }^{12}$ Ante la serie de eventos de diversa naturaleza experimentados en México y el resto de Latinoamérica en años recientes, cabría preguntarnos con capacidad de autocrítica, ¿qué tanto hemos avanzado en la región en cada uno de esos rubros?, ¿qué tipo de reformas y de cambios en las estructuras y en los sistemas de gestión de los aparatos gubernamentales se precisan para acceder a tales condiciones? ${ }^{13}$ Paradójicamente este sí es un fenómeno muy observable en las urbes latinoamericanas, donde conviven, junto a grandes cinturones de pobreza, colonias ricas donde la ostentación insultante, pareciera una premisa fundamental.

${ }^{14}$ Como señala Víctor L. Urquidi (2000: 135), el objetivo a largo plazo para la sociedad global, lo constituye el concepto de desarrollo sustentable y equitativo. En esa obra se plantea que así como la globalización ofrece riesgos, éstos se pueden ver superados por las oportunidades que incorpora para los países en desarrollo.
} 
Economía Informa núm. 391 marzo - abril • 2015

se trata de un fenómeno incompleto, parcial y desbalanceado; con avances vertiginosos en algunos campos, pero lentos en otros. Para ilustrar, según su propia apreciación, la muy relativa importancia del fenómeno analizado, apunta que el comercio y la inversión extranjera son considerablemente menores que el comercio interno y la inversión nacional.

Sin embargo, el autor no desconoce la compleja naturaleza de la globalización, su utilidad, así como la variedad de rasgos y significados que le son atribuidos. Por ejemplo, agrega Ffrench-Davis, muchos autores destacan sus componentes económicos, mientras que otros observan como contenidos dominantes los políticos o culturales; asimismo, mientras que generalmente la globalización es vinculada a acciones con tendencias a la convergencia y la homogeneidad, para otros investigadores está asociada a fenómenos de exclusión. Agrega que hay formas activas e intencionales de insertarse en la economía global que facilitan el fortalecimiento de la integración y el desarrollo nacional, y que la globalización, como mecanismo para potenciar los flujos comerciales, así como de inversión y tecnología, ha permitido el crecimiento y modernización de los sectores productivos de muchas economías en el mundo.

Ffrench-Davis indica acertadamente que los países subdesarrollados deben lograr una mejor inserción en la economía internacional, pero que esto no constituye una panacea, ya que vendría a resolver sólo parte del problema, sólo una fracción de la actividad económica. Por tanto, para este autor las políticas públicas y los mercados nacionales continuarán ocupando un espacio fundamental en la agenda política. Agrega que no existe una única receta aplicable en todo tiempo y en todo lugar, y que las políticas públicas continúan teniendo una base predominantemente nacional, en donde las diferencias entre las naciones continúan siendo muy relevantes. ${ }^{15}$ Remata observando que los países de América Latina se han distinguido por una excesiva uniformidad en la implementación de planes y programas de modernización y desarrollo (habría que agregar la clara tendencia o predilección por la importación acrítica de teorías y modelos), por ignorar el nivel del subdesarrollo de los mercados nacionales y por abrir las relaciones económicas hacia el exterior sin evaluar su impacto en las economías internas.

${ }^{15}$ Esta argumentación se inserta claramente en lo que muchos autores identifican como estilo nacional de generar políticas públicas, donde las variables que explican su hechura están representadas básicamente por la historia y la cultura del país; contrario al estilo ecológico de hacer políticas públicas, donde el contexto económico y político presente explica el tipo de política. Para profundizar en esta temática ver, por ejemplo, la obra de Méndez (1995: 4). 
Igualmente, para Ffrench-Davis la globalización se caracteriza por el incremento exponencial en la capacidad de las organizaciones privadas para fragmentar geográficamente los procesos de producción. ${ }^{16}$ Además, el investigador apunta que la globalización es un fenómeno heterogéneo, ya que mientras ciertos mercados muestran una clara movilidad e integración, como los financieros, en otros sectores como la construcción de infraestructura, la movilidad en el empleo o la seguridad social, los estilos nacionales prevalecen como características dominantes. Por consiguiente, agrega, la mayoría de las economías nacionales continúa orientada al mercado interno, lo que demuestra diciendo que el mundo exporta entre 15 y $20 \%$ de lo que produce al año, es decir, entre 80 y $85 \%$ del pIв mundial no se comercia internacionalmente; mientras que en lo que respecta al rango de ese 15 a 20\% de la producción que sí es exportada, el flujo es intensivo básicamente en el ámbito regional. No obstante, la afirmación precedente (respecto al comercio regional) no siempre se presenta en la realidad, como en Latinoamérica, donde sólo una quinta parte de su comercio se registra entre los países que la conforman.

De acuerdo a lo anterior, para Ffrench-Davis las exportaciones intrarregionales tienen mayor potencial para generar desarrollo económico y social, apoyando con esto el modelo de desarrollo endógeno, que ha de provenir del propio interior de las economías nacionales, como bien lo subraya. Además, en nuestra región, continúa, la calidad y diversificación de las exportaciones ha sido baja, por lo que la tarea pendiente consiste en desarrollar las condiciones para una mejor inserción en el comercio mundial. Lo anterior implica, recuerda Ffrench-Davis, inversión pública y privada con mayor perfil social, desarrollar los mercados de trabajo, tecnología y capital, así como la creación de un ambiente macroeconómico estable (condición esta última que generalmente no se ha presentado en el grueso de los países de nuestra región, donde ha imperado un desaseo en las finanzas públicas).

Otro dato que desmitifica la relevancia de la globalización es proporcionado por Ffrench-Davis: a pesar de que la inversión extranjera directa ha crecido más rápido que la producción, su contribución continúa siendo marginal respecto al total, ya que cerca de $90 \%$ de la inversión en el mundo se realiza con capitales nacionales, más bien, lo que se ha extendido añade el autor, son los flujos financieros. En este orden y de acuerdo al mismo investigador, la

\footnotetext{
${ }^{16}$ Como lo indica Hill (2011), esto se observa claramente en la elaboración de ropa, automóviles, calzado, artículos electrónicos e incluso en la prestación de diversos servicios, donde ciertos componentes se fabrican en diversos países pero el ensamble final, etiquetado, distribución o prestación puede ocurrir en otro muy distante.
} 
Economía Informa núm. 391 marzo - abril • 2015

captación de inversión extranjera depende de la capacidad de las economías locales de ofrecer ventajas específicas que alienten el ambiente industrial de una determinada región. Para él, a diferencia de lo apuntado por Stiglitz, los organismos multilaterales, a pesar de que se puede estar en desacuerdo con sus decisiones, han jugado un papel relevante en el proceso de desarrollo económico y han ayudado a solventar situaciones de crisis; en vez de su eliminación, recomienda mejorar su desempeño y su "desideologización".

Una de las lecciones que destaca el autor es que el proceso de globalización no es uniforme, y que las heterogeneidades que existen no son las más favorables para el desarrollo. Sin embargo, añade que en la práctica la disciplina macroeconómica impuesta por los mercados financieros es menor de lo que indican las posiciones extremas, por lo que incluso en áreas vinculadas al proceso de globalización financiera, las autoridades locales mantienen suficientes grados de libertad. Además, de acuerdo a Ffrench-Davis, a pesar de las tendencias a la homogeneización de la producción, las economías domésticas continúan mostrando fuertes rasgos intrínsecos respecto a la organización de los mercados y las relaciones de éstos con el Estado y la sociedad civil. ${ }^{17}$ Es decir, si bien para el autor la globalización tiene un efecto importante sobre los mercados y la efectividad de las políticas públicas locales, las autoridades nacionales mantienen diversos grados de libertad respecto al diseño, hechura e implantación de las políticas públicas, además de que éstas no obedecen a una sola fórmula válida en todo tiempo y lugar como recién se apuntó.

Ante el escenario de poco o nulo crecimiento (por lo tanto también de desarrollo) económico experimentado en América Latina y aunque pareciera una obviedad, Ffrench-Davis plantea que es necesario un mayor incremento del piB. Partiendo de que a pesar de que prevalece un amplio descontento con las reformas basadas en la economía de mercado que se han implantado en la región, las que han incorporado un enfoque demasiado economicista,

\footnotetext{
${ }^{17}$ Esto se puede constatar con la peculiar manera en que en México se conforman los mercados, ya que por ejemplo, a pesar de la existencia de un organismo federal encargado del combate de los monopolios, el esquema de mercados monopólicos u oligopólicos es el que prevalece (telefonía doméstica, cerveza, cemento, televisión, acero, vidrio, petróleo, electricidad, etc.). Esto se explica fundamentalmente (reconociendo no obstante que encontramos avances en la materia en diversos sectores) por patrones internos e históricos de carácter cultural, político (por ejemplo, el monopolio del poder político en un partido y en una persona, el Presidente de la República, fue una constante durante décadas), social y organizacional. Para una revisión de las tendencias de opinión pública respecto al cambio de modelo económico (donde la apertura de los mercados estaría vinculada a la modernización económica) ver la obra de Beltrán, Castaños, Del Pozo, Flores y Meyenberg (1996). Si se quiere profundizar en las relaciones entre las culturas nacionales y la organización social, política y administrativa, consultar la obra de Hofstede (1991).
} 
olvidando que el fin es mejorar las condiciones de vida de la población no la mera aplicación del instrumento en sí, el autor propone una salida pragmática a esta situación, sin cargas ideológicas. Desechando opciones como "el borrón y cuenta nueva" que originarían la experimentación con modelos alternativos, lo que Ffrench-Davis propone es, dentro de las variantes de la economía de mercado, reformar las reformas, de tal forma que se corrijan sus fallas y se fortalezcan sus atributos. Es decir, que prevalezca un sentido de aplicabilidad, conociendo cómo operan realmente los mercados y realizando cambios funcionales, adecuados para esos mercados.

Ffrench-Davis reconoce adecuadamente que esa nueva reforma no se podrá originar únicamente con simples buenos deseos, para crecer sostenidamente, advierte, se requiere crear mayor capacidad de producción, mayor cantidad y calidad de capital y de trabajo, la permanente incorporación de nuevas tecnologías, además de un equilibrio macroeconómico sostenido. Como complemento a lo anterior, agrega, es necesario solidificar nuestras exportaciones mediante la eliminación de sus dos principales defectos: la concentración en recursos naturales con valor agregado muy reducido y la inestabilidad de sus precios internacionales.

La conclusión planteada por este autor es que los Estados nacionales no están irremediablemente condenados a la impotencia o a la aceptación pasiva de los efectos de la globalización; si así fuera, la gobernabilidad sufriría un desgaste letal, originando más escenarios de conflicto e inestabilidad de los que ya hemos experimentado en América Latina en los últimos lustros. Además, continúa el autor, la globalización ofrece oportunidades como la posibilidad de mejorar las condiciones de acceso a los mercados y la creación de nuevas opciones para los agentes económicos participantes, a medida que se alienta la competencia y se desarticulan los monopolios establecidos. Sin embargo, añade que estas oportunidades son sólo potenciales (meros proyectos) y no se presentan en forma homogénea entre los países; no existiendo garantía de que a pesar del alto costo de generar políticas insostenibles en el mediano y largo plazo, las políticas públicas que se implanten serán superiores a las aplicadas en épocas pasadas. ${ }^{18}$

\footnotetext{
${ }^{18}$ Varios autores explican este hecho con variables analizadas por la Teoría de la Organización. Por ejemplo, Hall (1996) plantea que la rigidez y conservadurismo de las burocracias gubernamentales (incluso en la empresa privada) dificulta la implantación de nuevos programas y políticas públicas. Por su parte, Pfeffer (2000) acuña el término de isomorfismo organizacional, el cual explica por qué las formas organizacionales dominantes generalmente son muy parecidas. A su vez, este isomorfismo es originado por fuerzas coercitivas del medio ambiente (como las reglamentaciones) o la imitación institucional.
} 
Finalmente, Ffrench-Davis sugiere con acierto que este nuevo escenario plantea retos muy evidentes al Estado, el cual requiere ser transformado para estar a la altura de los actuales desafíos contextuales. Entre los rasgos exigidos a este nuevo modelo de Estado figuran el que se vuelva más ágil en términos administrativos y organizacionales, más profesionalizado, capaz de interpretar y atender las demandas de la población, además de preparado para emplear de manera eficiente el margen de discrecionalidad en las políticas públicas. ${ }^{19}$

\section{Conclusiones}

En función de lo revisado primeramente debemos resaltar que en los últimos lustros Latinoamérica experimenta una nada despreciable mejoría en sus indicadores de desarrollo económico, además de que la percepción de la ciudadanía respecto a la economía, salvo contadas excepciones, es favorable. Lo anterior coincide con la implementación del modelo de economía de mercado en el grueso de nuestras naciones, por lo que es procedente suponer algún tipo de correlación positiva entre ambos fenómenos (además, como se vio, la gente cree en este modelo económico). No obstante, en la mayoría de nuestras naciones no hay una sensación de progreso económico, pero sí hay una alta percepción de corrupción y, de manera simultánea, de desigualdad social y política.

Lo anterior permite responder nuestra pregunta central de investigación a la vez que corrobora nuestra hipótesis de trabajo: La evidencia sugiere que la globalización sí es una variable fundamental para entender nuestro desarrollo social y económico, pero a la vez existen otras variables explicativas sustantivas. Así, gran parte de los aprietos experimentados en Latinoamérica obedecen a la construcción de organismos y sistemas de gobierno ineficaces, a la débil presencia de arreglos institucionales más racionales que permitan generar mejores políticas públicas, $y$, fundamentalmente, a la generación se aparatos públicos muy corruptos (con la excepción de Chile y Uruguay), como lo corroboran los planteamientos de Stiglitz y de Ffrench-Davis.

Tal como apunta el propio Stiglitz, los países que más provecho han obtenido de la globalización han sido los que tomaron las riendas de su propio destino y reconocieron el importante rol que puede cumplir el Estado en el desarrollo, sin esperar que el mercado solucionara todos los rezagos. Por

\footnotetext{
${ }^{19}$ Ciertamente las medidas planteadas por Ffrench-Davis no por ser adecuadas son novedosas. Para profundizar en las medidas consideradas como útiles para reformar a nuestros Estados se recomienda consultar el trabajo de Kliksberg (1989).
} 
consiguiente, los países que conforman nuestro subcontinente deben asumir la responsabilidad de su propio desarrollo y bienestar; éste no vendrá por arte de magia del exterior, más bien habrá que construirlo internamente a partir de una visión de desarrollo endógeno, atento a las señales del exterior. Davis agregaría que el Estado en América Latina requiere ser reformado para responder a las exigencias de la modernidad y se vuelva más eficiente y profesional.

Este trabajo se atreve a proponer el dejar atrás posturas extremas o sobre ideologizadas, las cuales poco aportan para solucionar nuestras agudas deficiencias. En este sentido, la realidad obliga a que al fenómeno de la globalización sea observado desde todos los ángulos que nos ofrece, considerando también que tiene efectos positivos y negativos, que plantea riesgos y oportunidades a la vez, en suma, que no representa ni la maldición ni la panacea. Al respecto y como antes se expuso, Ffrench-Davis desmitifica varias de las suposiciones en torno a este fenómeno y plantea que la globalización se construye a partir de lo local. Parece importante aplicar en América Latina la recomendación del mismo autor en cuanto a darle impulso a los mercados, aumentar la productividad, el equilibrio macroeconómico y las exportaciones, lo que junto a la reconversión del Estado, constituye una alternativa de solución. Reformar a las reformas diría él, es decir, a partir del modelo sustentado en el mercado, mejorar su estructura y su dirección.

En esta delicada tarea resultará conveniente respaldar la propuesta que formulara Stiglitz, en el sentido de que al Estado y al mercado hay que verlos como socios. El Estado haciendo los arreglos necesarios para el buen funcionamiento de los mercados, de tal forma que éstos permitan dinamizar nuestras economías y la creación de empleos, pero sin renunciar a su obligación de supervisarlos y regularlos. Como bien dice este autor, tratar de acceder a una globalización con rostro más humano. En tal sentido, este ensayo coincide no sólo con Stiglitz y con Davis, también con Boron, cuando señalan que el ámbito público o estatal no es por naturaleza ineficiente y que el sector privado por definición es eficiente. Ambos pueden alcanzar la excelencia si hay una buena delimitación de objetivos, estrategias, funciones y programas; el escenario global exige que así sea.

Ante tal escenario, es importante evitar caer en los fundamentalismos. Ya sea en el fundamentalismo del mercado, que propone que existe un solo grupo de políticas económicas (pro mercado) correctas, o en aquel que achaca la responsabilidad de todos los males que aquejan a nuestra región a la economía de mercado y la globalización. Busquemos una posición más objetiva e intermedia y comprendamos que ni los mercados ni la empresa privada son del 
todo eficientes, y que ni los Estados ni políticas públicas promuevan siempre la ineficiencia.

Lo que sí debe ser reconocido es que gran parte de la desventajosa situación vivida en Latinoamérica en materia de crecimiento económico, distribución de la riqueza, generación de empleos, corrupción, inseguridad y narcotráfico, obedece a que no hemos construido las instituciones públicas lo suficientemente sólidas para su superación, independientemente de los vaivenes y fuerzas provenientes del exterior. Es decir y como lo sugiere Ricardo Ffrench-Davis, las respuestas a las interrogantes en torno a nuestro subdesarrollo habrán de encontrarse, nuevamente, mirando hacia nosotros mismos, más que hacia afuera. La evidencia indica que ese reconocimiento debe ser el punto de partida para tratar de generar un desarrollo más sólido para la región en todos los órdenes de realización humana.

\section{Bibliografía}

Banco Mundial (2015), "Banco de datos mundial. Indicadores de desarrollo mundial" (consultado el 04 de febrero de 2015), disponible en: http://datos.bancomundial.org/indicador/NY.GDP.PCAP.CD.

Beltrán, Ulises et al. (1996), Los mexicanos de los noventa. México, Instituto de Investigaciones Sociales, UNAM.

Boron, Atilio (2002), Imperio e Imperialismo, Buenos Aires, Consejo Latinoamericano de Ciencias Sociales.

Corporación Latinobarómetro (2010), Informe 2010. Santiago de Chile, Latinobarómetro.

Ffrench-Davis, Ricardo (2002), Reformas económicas, globalización y gobernabilidad en América Latina, Caracas, Fundación Desarrollo y Paz-Editorial Nueva Sociedad.

Hall, Richard (1996), Organizaciones, estructuras, procesos y resultados, México, Prentice Hall.

Hill, Charles (2011), Negocios internacionales. Competencia en el mercado global, México, McGraw-Hill Interamericana.

Hofstede, Geert (1991), Cultures and organizations. Software of the mind, Nueva York, McGraw-Hill International.

Kliksberg, Bernardo (1989), Gerencia pública en tiempos de incertidumbre, Madrid, INAP.

Kliksberg, Bernardo (2007), “¿Cómo avanzar la participación en América Latina, el continente más desigual?”, Revista del CLAD Reforma y Democracia, núm. 37, Caracas, CLAD, febrero, pp. 1-27. 
Koontz, Harold, Weihrich, Heinz y Cannice, Mark (2008), Administración. Una perspectiva global y empresarial, México, McGraw-Hill Interamericana.

Kroes, Rob (2002), Ciudadanía y Globalización. Madrid, Ediciones Cátedra.

Méndez, José L. (1995), Regiones y política industrial en Nuevo León, México, Westfalia del Rhin del Norte, Alemania y Pennsylvania, Estados Unidos, México, El Colegio de México.

Osborne, David y Gaebler, Ted (1992), Reinventing government, Nueva York, Addison Wesley.

Pfeffer, Jeffrey, (2000), Nuevos rumbos en la Teoría de la organización, México, Oxford University Press.

Rodríguez, Martín (1997), "Teorías y enfoques de desarrollo económico”, Papeles de población, núm. 13, México, Universidad Autónoma del Estado de México, enero-marzo, pp. 55-74.

Stiglitz, Joseph E. (2002), El malestar en la globalización, Buenos Aires, Alfaguara.

Transparencia Internacional España (2015). “Índice de Percepción de la Corrupción (IPC) 2014” (consultado el 04 de febrero de 2015), disponible en: <http://www. transparencia.org.es/IPC's/IPC_2014/Indice_IPC_2014.htm>.

Urquidi, Víctor L. (coord.) (2000), México en la globalización. Condiciones y requisitos para un desarrollo sustentable y equitativo, México, Fondo de Cultura Económica. 